\title{
RECENT TRENDS AND SPACE APPLICATIONS OF POLYIMIDES
}

\author{
RIKIO YOKOTA
}

\author{
Institute of Space and Astronautical Science(ISAS), Ministry of Education. \\ 3-3-1 Yoshinodai, Sagamihara-shi, Kanagawa, Japan 226 \\ Tel. +427-59-8056, Fax. +427-59-4251, e-mail; riki@pub.isas.ac.jp
}

\begin{abstract}
This paper presents the science and technology developments in the field of high performance aromatic polyimides and their composites in relation to space applications as follows; 1) Development of polyimides as an high performance materials 2) High performance polyimide film like materials for spacecraft applications such as the multilayer thermal blanket(MLI) and the flexible and/or rigid extendable structures, 3) Development of these heat resistant polyimide matrix resins for advanced composites. Finally, this paper describes the synthesis and properties of a newly developed, phenylethynyl-terminated novel asymmetric biphenyltetracarboxylic dianhydide (a-BPDA) derived polyimide matrix resin with high $\mathrm{Tg}$, good processability, and good toughness.

Keywords; space application, thermal stability, composite, asymmetric biphenyltetracarboxylic dianhydride, phenylethynyl-terminated polyimide
\end{abstract}

\section{Introduction}

Since the first commercial polyimide was introduced by $\mathrm{Du}$ Pont in the late $1960 \mathrm{~s}$, polyimide science and technology has been extremely developed relating in thousands of papers on these miracle polymeric materials[1-3]. During the early 1980 s, aromatic polyimides have been widely used in the microelectronics industry as dielectric and passivation layers because of their outstanding properties such as their low thermal expansion and high dimensional stability in addition to good planarization, good processability, excellent mechanical properties, and environmental stability. It is well known that Kapton polyimide consisting of PMDA and ODA was mainly used for special applications such as space and military requirements until the 1970s[1]. However, there is no standard definition for high performance polymers because the requirements and environments for different applications vary significantly. Polyimides are a special class, real high performance materials for use in advanced engineering applications for a wide industry. This paper describes the relationship between the
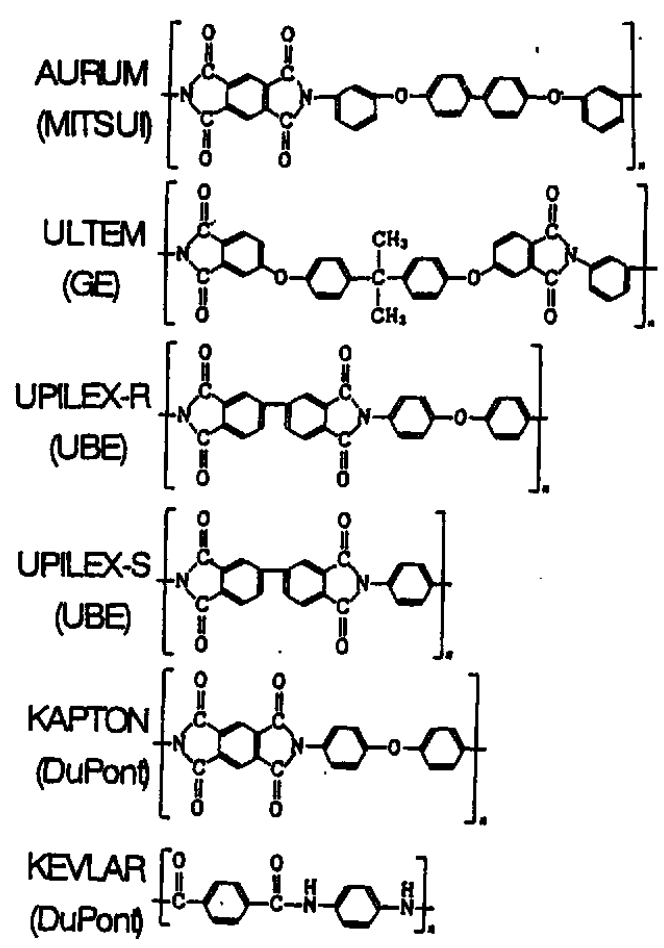

Figure 1. Chemical structures of commercially available, space application polyimide films. 
development of polyimide science and technology in relation to its aerospace applications. Finally, this paper presents the synthesis and property of a newly developed novel asymmetric biphenyl type polyimide (a-BPDA) derived from high temperature polyimide matrix resins with good processability and good toughness[9].

\section{Development of polyimides as high performance materials}

If we consider the progress in polyimide science and technology, it is divided into three steps in historical development as follows. 1) Aromatic polyimides having rigid, planar structures such as pyromellitimide and semiflexible biphenylimide were understood to favor the formation of high-order structures, resulting in excellent properties of high dimensional stability, low thermal expansion, wide range thermal stability and so on. 2) Functional polyimides having high optical transparency and/or low refractive index or low dielectric constant were developed significantly in the 1990 s. 3)From the engineering point of view, it has been pointed out that several novel thermoplastic polyimides are available or are being considered for individual engineering purposes.

In the case of 1), many books and papers have been published and it seems that the structureproperty relationship between chemical and high-order structures such as in-plane orientation, chain aggregation, intra- and intermolecular interaction in relation to polyimide film properties with processing conditions of the polyimide film is well understood[23]. In recent years, polyimides have been introduced more and more into the microelectronics industry. Low dielectric constant polyimide film is a quite important for applications involving interlayer dielectrics. The refractive index is also an important parameter for optical applications of polyimides. Colorless polyimide films were first reported by NASA for varying the molecular structure and reducing the electronic interaction between chromophoric sites for space applications [4]. They were composed of aromatic dianhydride and diamine monomers because they provide radiation resistivity in addition to less colored for use in space. On the contrary, most colorless polyimides for recent microelectronic applications are prepared by alicyclic dianhydride and/or alicyclic diamine, or fluorinated monomers, because they reduced the dielectric constant and the refractive index in addition to the property of low water absorption[5,6].

With regards to thermoplastic polyimides of step 3), Polyetherimide, ULTEM of GE was only a resin of an amorphous thermoplastic polyimide until the 1980s. However, the applications of polyimides ranged from aerospace to microelectric and wide industry, with various imide monomers being developed. As a result, so called molecular design on thermoplastic polyimides have been already completed, resulting in development of the new, medium class heat resistant thermoplastic polyimides such as AURUM of Mitsui [7] and PIXEO of KANEKA.

With regard to polyimide matrix resins for heat resistant composites, polyimides containing rigid heteroaromatic rings give poor processability even with the addition polymerization of oligoimide such as BMI and PMR-15. Because of the rigid primary structures, favoring the strong intermolecular ordered structure, it seems to be an

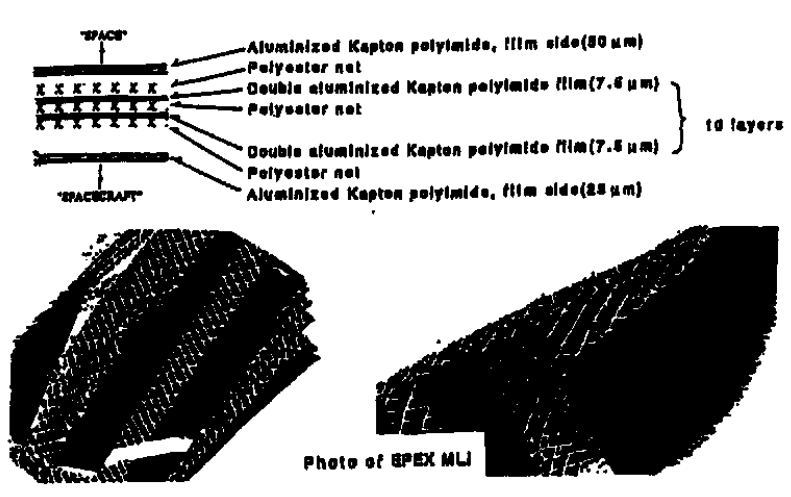

(b)

Figure 2 Configuration of aromatic polyimides MLI for Space Flyer Unit(SFU) 
intrinsic problem that aromatic polyimide can't proceed in molecular mobility beyond $\mathrm{Tg}$, resulting in a requirement of extremely severe processing condition for structural applications. However, we recently found the fact that asymmetric biphenyl type polyimides having a considerably inflected chain structure exhibited very curious viscoelestic behavior indicating the high $\mathrm{Tg}$ and large fall of the storage modulus, $\mathrm{E}^{\prime}$ above the $\operatorname{Tg}[8]$. Finally, this paper presents the synthesis and property of a newly developed novel asymmetric biphenyl type polyimide (a-BPDA) derived from high temperature polyimide matrix resins with good processability and good toughness[9].

\section{Aromatic polyimides for space applications}

\subsection{Multilayer thermal blanket(MLI)}

Figure 1 shows the chemical structures of commercially available aromatic polyimides for space applications[10]. With regard to the space application of the high performance polyimides in step 1), multilayer insulation such as the so called multilayer thermal blanket(MLI), is a typical example of aromatic polyimides for space applications. Spacecraft themselves and each instruments within are generally covered with the various metalized polymer films for controlling the temperature of the instruments in the spacecraft. Surface temperature of a metalized polymer film is a function of the ratio of solar absorptivity $(\alpha)$ to low temperature emissivity $(\varepsilon)$ of the film. At equilibrium, a low $\alpha / \varepsilon$ ratio provide a low surface temperature(high values provide a high surface temperature). Therefore, by choosing the proper film and the appropriate metal, it is possible to specify a thermal control surface within a wide range of $\alpha$ and $\varepsilon$ values. A passive (nonactive) thermal control system such as MLI significantly helps to maintain spacecraft systems and components at specified temperature limits. Kapton polyimide and Teflon films have long been accepted as the space stable insulating materials. Figure 2 shows the configuration of the aromatic polyimide MLI for SFU, which is the
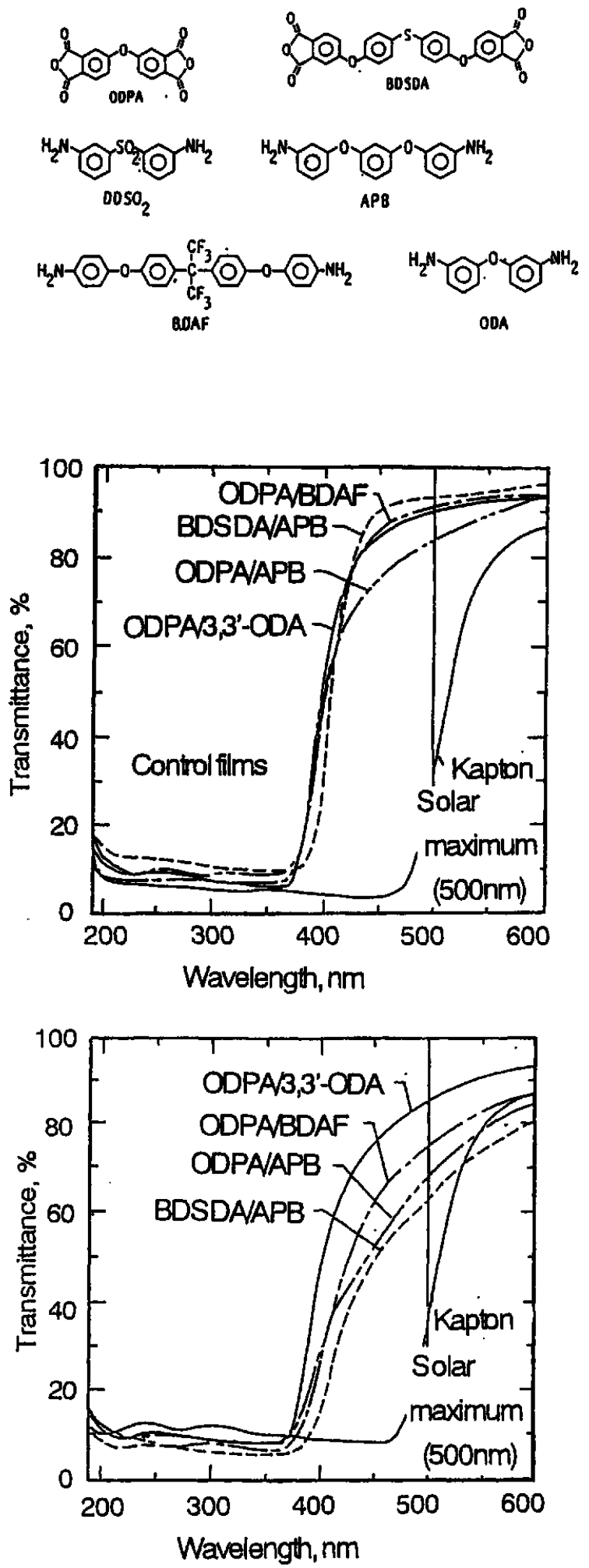

Figure 3. UV-vis spectra of controlled(a) and exposed(b) pale/colorless polyimide films and Kapton. 

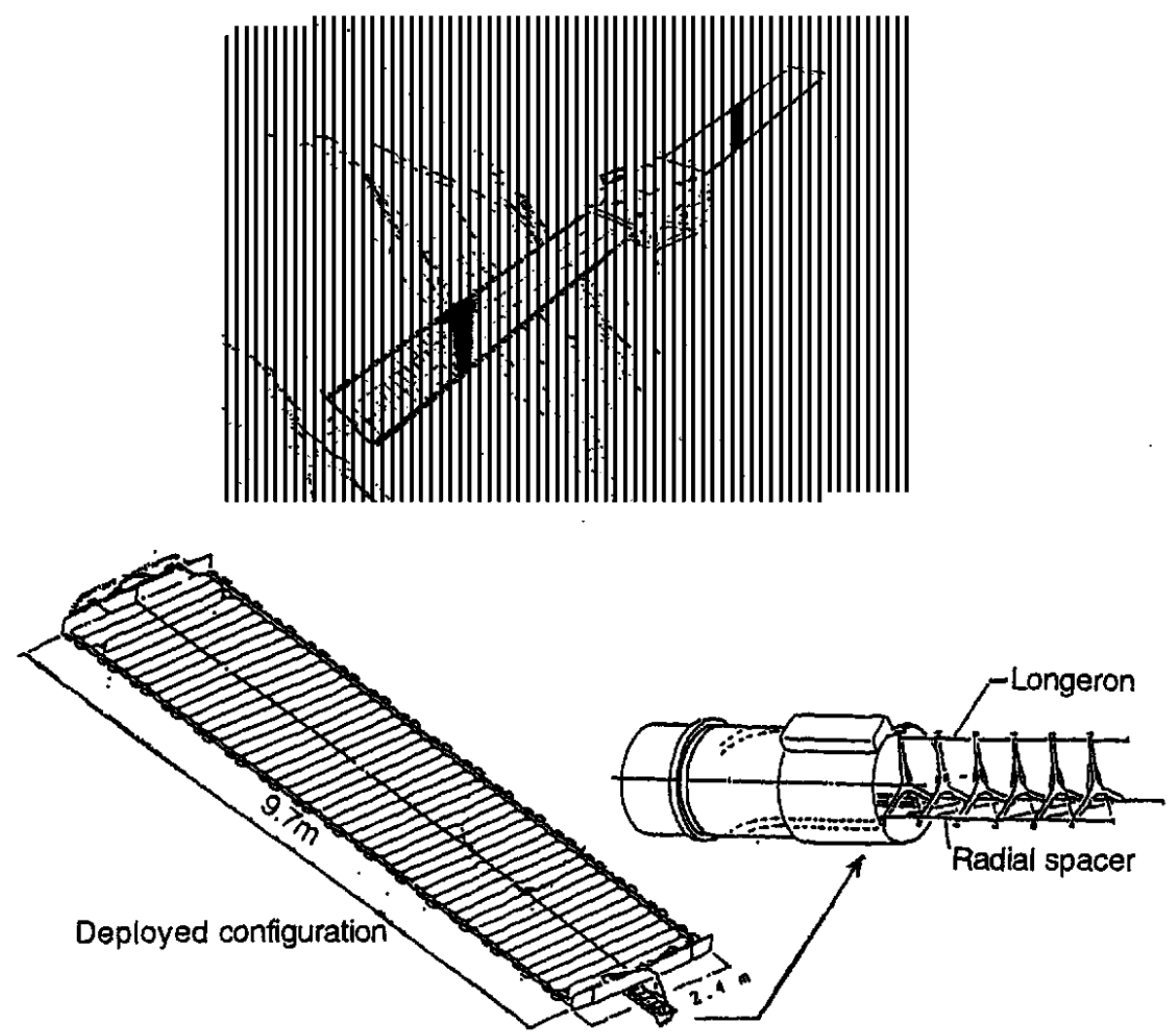

Figure 4. Illustrated SFU spacecraft and deployed configuration of its solar array with the extendable mast.

first Japanese spacecraft retrieved by the Space Shuttle in 1995. The outer layer uses $50 \mu$ m thick aluminized KAPTON with 11 layers of double aluminized thin KAPTON films. The ASCA spacecraft that is a powerful X-ray observatory satellite of ISAS launched in the 1993. Because the maximum allowable fluctuation of the focal point is $0.5 \mathrm{~mm}$, the surface of $3.4 \mathrm{~m}$ long, high precision Extendible Optical Bench (made of CFRP)was covered with a UPILEX-R polyimide MLI. UPILEX-R consisting of a biphenylimide with 4,4'-biphenyl ether, possesses a higher optical transparency of the film compared with that of pyromellitimide Kapton. This is an advantage for the system due to the low absorptivity. A new MLI covering over $90 \%$ of the outer layer covering of the spacecraft ASCA is composed by 2 outer layers of aluminized $25 \mu$ $m$ UPILEX-R and 5 layers of double-aluminized $12 \mu \mathrm{m}$ polyester films with a separator net. Until 1987, Kapton MLI was the only MLI for the high temperature areas of spacecraft and ASCA is the first satellite completely covered by an another polyimide MLI. Colorless polyimide films was first reported by NASA for varying the molecular structure of reducing the electronic interaction between chromophoric centers for space applications. Because of the requirement of radiation resistivity in space, they were prepared by aromatic dianhydride and diamine monomers[11,12]. Figure $3 a$ and $3 b$ show the UV-vis spectra of controlled and exposed pale/colorless polyimide films and Kapton[4].

\subsection{Flexible and/or rigid extendable structures}

\subsubsection{A flexible solar array}

A flexible solar array is an attractive example of combining an extendable advanced composite with aromatic polyimide film. Japan's first spacecraft SFU deployed the two large flexible solar arrays in low earth orbit(LEO). Due to the high $\mathrm{Tg}$ and outstanding mechanical properties, even in very low temperature environments, aromatic polyimide is the most successful, widely 
used polymeric material in space [10]. Until ten years ago, a spacecraft was usually equipped with a rigid type power generator(solar paddle). However, as a spacecraft becomes larger, it requires much more electric power. It is thought that a flexible solar array is the most attractive way for power generation in space. Figure 4 illustrates the SFU spacecraft and the array configuration with the extendable mast. Each deployed solar array is $2.4 \mathrm{~m}$ wide and $9.7 \mathrm{~m}$ long. The array is composed of two assembly boards and the mast canister. The extendable/retractable mast is a continual coilable mast consisting of three GFRP spring rods(longerons) and radial spacers. The main source of its spring force is generated by the bending strain energy of the GFRP longerons. The radial spacers were made of molded UPILEX-R, because of its excellent space environmental stability without creep behavior originating from the high intermolecular interaction of benzoimide rings. Therefore, no mechanical backlash exists(since there are no pin-joint hinges), resulting in high dimensional stability. Each array blanket consists of 48 hinged low C.T.E UPILEX-S polyimide films, whose size is $202 \mathrm{~mm}$ wide and $2,400 \mathrm{~mm}$ long. About 27,000 solar cells are mounted on two array blankets and generate
$3.0 \mathrm{~kW}$ of power. 100 micrometer thickness silicon cells with 100 micrometer cover glass are adhered by S-691-RTV silicon type adhesive on the polyimide panels[13].

\subsubsection{Development of a large deployable antenna}

Development of a large deployable antenna on Muses-B is another example of advanced technology in space[14]. Figure 5 illustrates the Muses-B antenna launched in 1997 to be used aboard the satellite for Space-VLBI(Very Long Baseline Interferometry). A $10 \mathrm{~m}$ diameter, parabolic antenna with mesh surface was deployed with steps extending the six extendable masts in LEO. This incredibly complex system consists of 6,000 fine cables of high modulus Kevlar 149 aramid covered by a CONEX aramid net. In order to achieve high surface accuracy, a cable must precisely keep its length without creep under a tension field in space. The high strength Kevlar 149 cable exhibits very little elongation when stressed and has negative C.T.E over a wide range of temperatures. Because the lengths and tension force of the cable was critical, the tension force of each extendable mast was strictly controlled by the tensioners on the top of the masts. It was the first

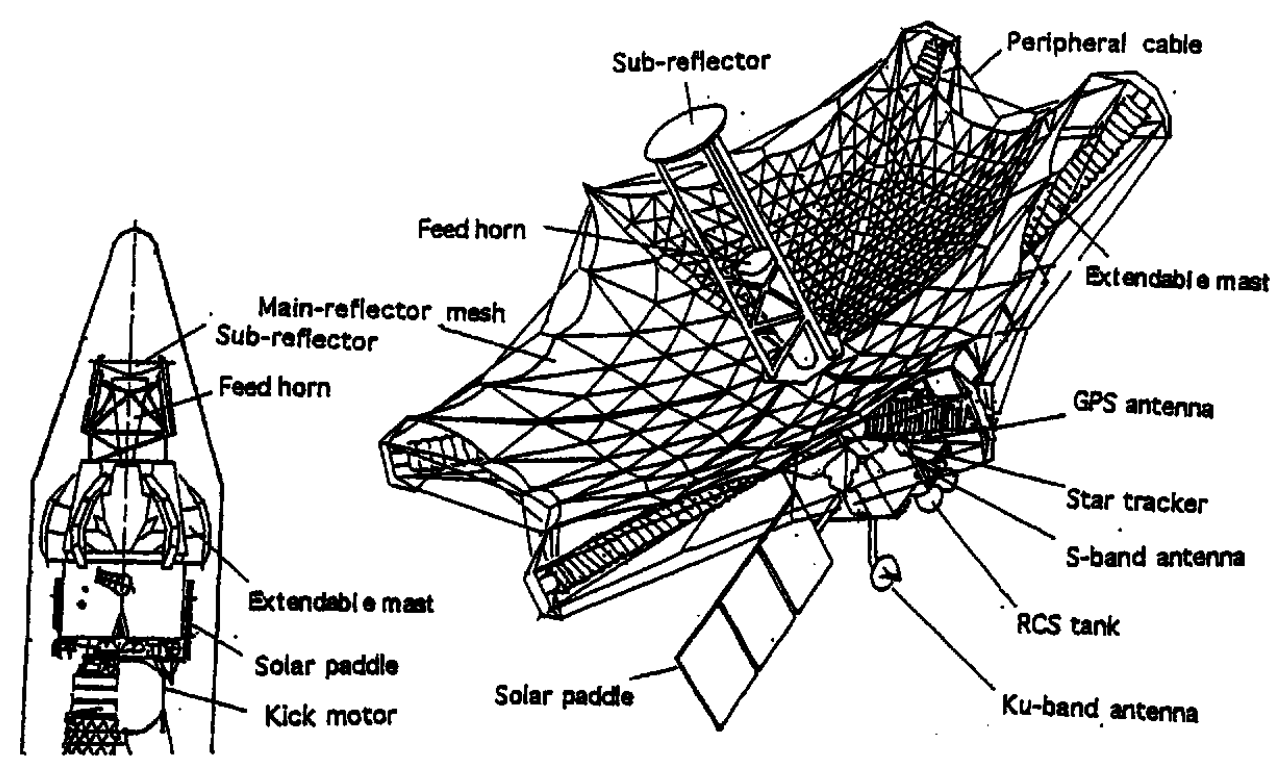

Figure 5. Configuration of MUSES-B antenna; a) stowed in the nose faring b) deployed. 


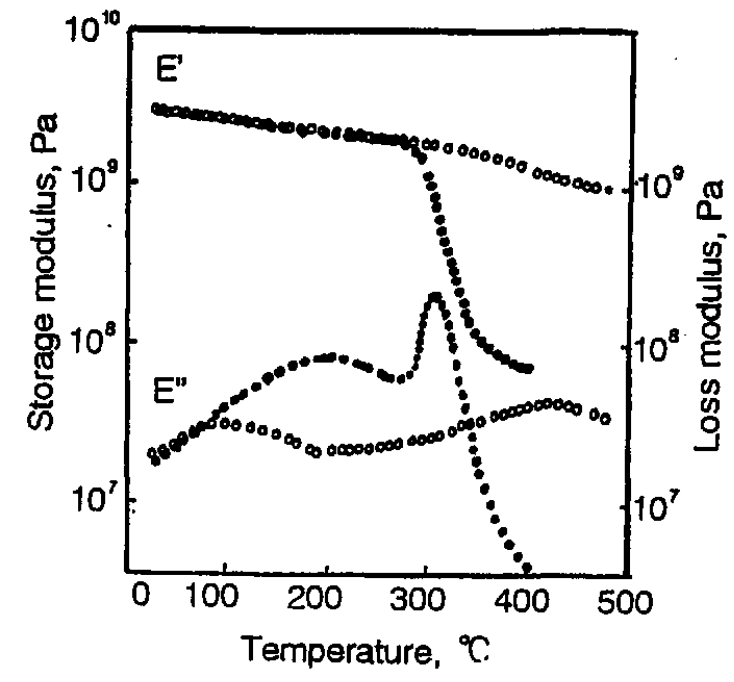

Figure 6. Temperature dependence of dynamic tensile property for Kapton(o) and UPILEX-R( () type polyimide films.

application of high performance organic fibers for a large deployable parabolic antenna surface in space.

\section{Development of the heat resistant polyimide matrix resins}

Aromatic polyimides are widely used in spacecraft materials, due to their excellent space environmental stability in addition to high thermal, mechanical, and electrical properties[3].

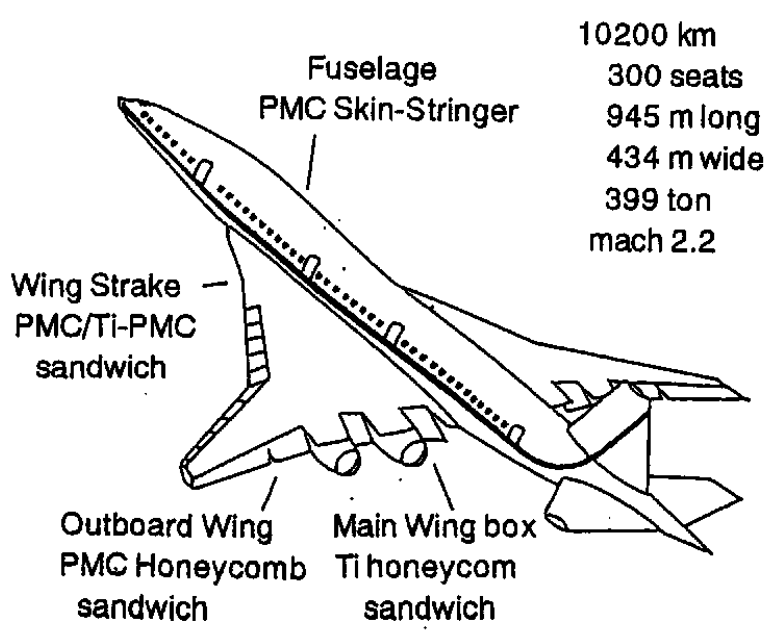

Figure 7. Materials and structures of next generation super sonic airplane.
Polyimide films are now indispensable as film type materials for a passive thermal control system of spacecraft as well as the only material for flexible solar array as mentioned previously. However, it is well known that the outstanding properties of polyimides are based not only on the rigid, planar

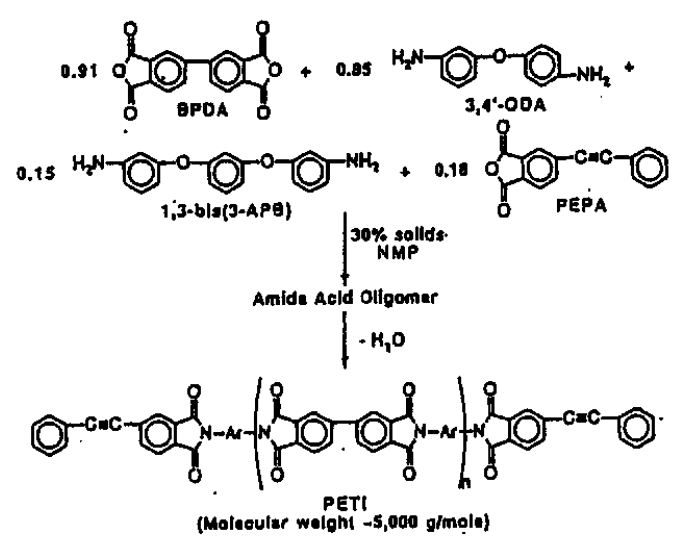

Figure 8. Synthetic scheme of PETI-5.

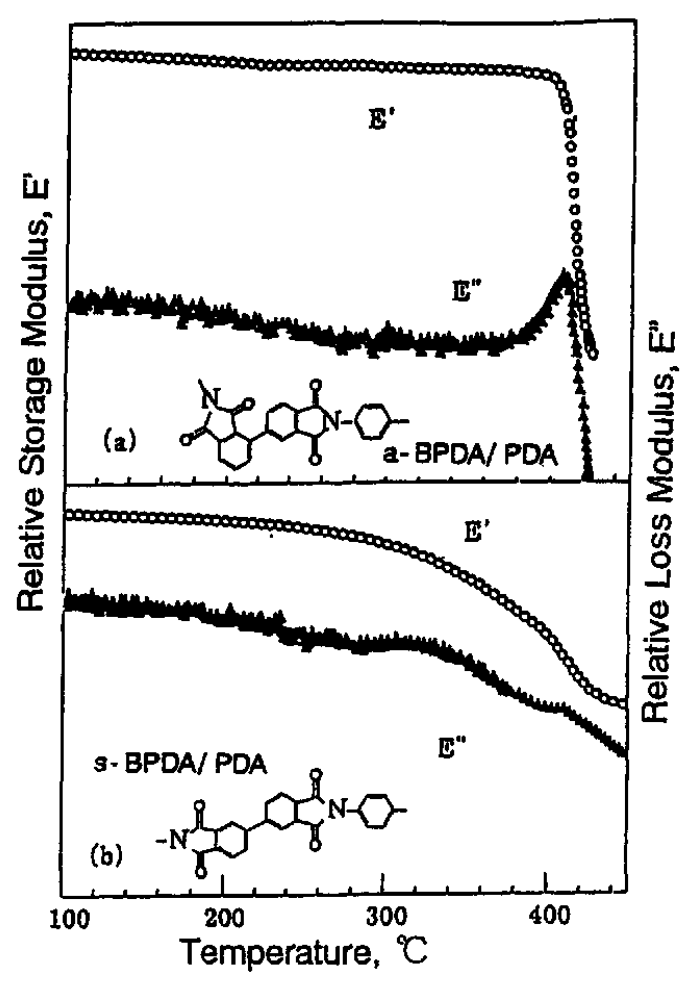

Figure 9. Temperature dependence of dynamic tensile property for isomeric biphenyl type polyimide films. 
structure of benzoimide moiety, but also on the formation of high-order structures, even beyond $\mathrm{Tg}$. As a result, most of aromatic polyimides can' $t$ proceed molecular mobility above $\mathrm{Tg}$, resulting in extremely severe processing conditions for molding materials. Figure 6 is an example of the temperature dependence of dynamic viscoelastic behavior for Kapton and UPILEX-R type aromatic polyimide films. It exhibits very low molecular mobility and high melt viscosity of a flexible later type polyimide. Although, many addition type polyimide resins such as bismaleimide and PMR-15 containing rigid heteroaromatic rings have been developed in the past 20 years, the heat resistant composites prepared from these addition type polyimide oligomers require severe processing conditions [3,15]. Recently, the next generation of super sonic transports(SST) have been being planned by international project shown in Figure $7[18]$. The structure of a SST requires long term durability of the materials in cruise environments with temperature ranging from $-55^{\circ} \mathrm{C}$ to $160^{\circ} \mathrm{C}$. Therefore, many fundamental studies have been conducted for investigating micro-cracking, fatigue, and oxidation on advanced polyimide composites. PETI-5 which was developed by NASA is a lightly crosslinked thermoplastic
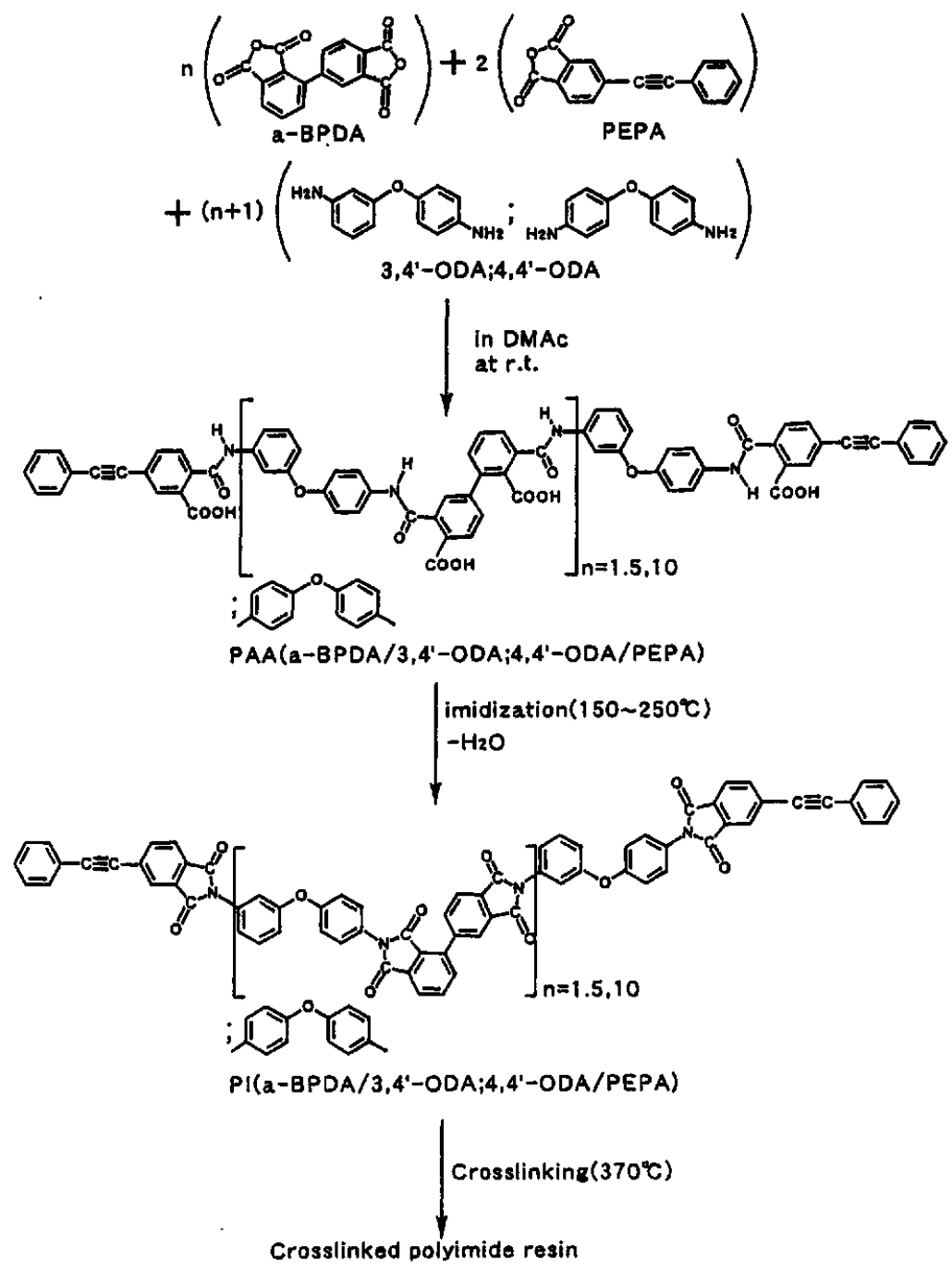

Figure 10. Synthesis of a phenylethynyl-terminated, asymmetric biphenyltetracarboxylic dianhydride (a-BPDA) derived polyimide resin 
shown in Figure 8[16]. Due to the medium size molecular weight, Mc=5.000 between crosslinking sites, cured PETI-5 exhibits more than $10 \%$ elongation before the film break resulting in a $\mathrm{Tg}=270{ }^{\circ} \mathrm{C}$ and a fracture toughness of $\mathrm{G}_{\mathrm{ic}}=4795\left(\mathrm{Jm}^{-2}\right)$. In order to improve the fracture toughness, the cross-link density must be reduced. It is known that the processability of the resin is greatly affected by the viscoelastic property in the rubbery region above $\mathrm{Tg}$ of the main chain between the crosslinking sites. Therefore, the requirement of high thermostability in addition to good processability and good toughness seems to be contradictory to the development of heat resistant polyimide matrix resins.

We recently discovered that the asymmetric biphenyl type polyimide, a-BPDA/PDA, having a considerably inflected chain structure, exhibited very curious viscoelestic behavior resulting in a high $\mathrm{Tg}$ and a large steep decrease in the storage modulus, E' above the Tg. Figure 9 is shows the temperature dependence of dynamic tensile properties for isomeric biphenyl type polyimide films. The a-BPDA/PDA annealed at $400^{\circ} \mathrm{C}$ shows a higher $\mathrm{Tg}$ than the s-BPDA/PDA treated under the same conditions $(8)$. The difference between the extent of the storage modulus, E' decrease at the $\mathrm{Tg}$ for both polyimides is attributed to the difference in the intensity of intermolecular interactions of two isomeric BPDA molecules, suggesting the improvement of the processability of a-BPDA derived polyimide resins.

As shown in Figure 10, we successfully developed novel a-BPDA derived high temperature polyimide matrix resins with good processability and good toughness[9].

\section{References}

1) Heat Resistant Polymers, Technologically Useful materials, ed. by J.P.Critchley, G.J.Knight, and W.W.Wright, (1983) Plenum

2) Polyimides Fundamentals and Applications ed.,by K.L.Mittal and M.K.Ghosh (1995) Elsevier 3) R.Yokota, Structure and design of photosensitive polyimides, ed. by K. Horie \& T.Yamashita, chap. 3, TECHNOMIC 1996

4) A.K.St.Clair et.al, SAMPE J, July/Aug. p28 1985

5) S, Ando、 Recent Progress on Polyimides 1995、 p11, ed.by M. Hasegawa \& R. Yokota、Reitec CO、 1996

6) Q.Li, K.Horie, and R.Yokota, J. Photopolymer Sci. and tech., vol.10, No.1 (1998) p49

7) M.Ohta, J.New Materials, (1990) p6, Nippon Kogyou Shuppan

8) M.Hasegawa, R Yokota, N. Sensui, and Y. Shindo, Macromolecules, vol.32 No.2 (1999) p387

9) R. Yokota et al, Proceedings of 50th Int. Astronautical Congress, 1999.10, Amsterdam in press.

10) R. Yokota, Proceedings of 1st Oxford Kobe Materials : Aerospace Materials, St Catherine's College Kobe Institute, 1998.9

11) R.Yokota, Proceedings of 7th Int. Conf. on Materials in a Space Environment, p (1997) ESA/ONERA

12) NASA CP3134 Partl-3 (1992) 、CP3164 Partl-2 (1992)、 CP3194 Partl-3 (1993)

13) Y.Shibayama et.al, Proceedings of the Eur. Space power Conf., Florence, p135 Italy 1991

14) T.Takano et al, ISTS-96-e-18, May 1996, Japan

15) T.T Serafini, Polyimides; Synthesis, characterization, and applications, ed. by K.L.Mittal, vol.2 p957 1984 Plenum, NY

16) T.H.Hou et al., J. of Composite Mats., vol.30, No.1, p109 (1996)

17) R. Yokota et al, Proceedings of 7th Int. Conf. on Materials in a Space Environment, Toulouse, France, June 1997, p ESA/ONERA

18)1997 Special Report on the next Generation SST, (1997) Japan Space and Aeronautical Industry 